\title{
Surto de leishmaniose tegumentar americana em Canoa, Santo Amaro, Bahia, Brasil
}

\author{
An outbreak of American cutaneous leishmaniasis in Canoa, \\ Santo Amaro, Bahia, Brazil \\ Ivonise Follador, Cibele Araujo, Maria Amélia Cardoso, José Tavares-Neto, Aldina Barral, \\ José Carlos Miranda, Achiléa Bittencourt e Edgar M. Carvalho
}

\begin{abstract}
Resumo Em 1993, um surto leishmaniose tegumentar americana (LTA) foi detectado no povoado rural de Canoa, município de Santo Amaro, Bahia. Um estudo observacional prospectivo delineouse, com objetivo de determinar as taxas de freqüência e caracterizar clinicamente a doença. Foram acompanhados 555 indivíduos, registrando-se 29 casos de LTA, 11 casos sugestivos de LTA pregressa e 529 sadios. Desses 529 sadios, 65 apresentaram reação de Montenegro positiva sem qualquer evidência presente ou passada de doença. A prevalência de LTA no período de estudo foi de 5,2\% (29/555). A leishmania envolvida foi caracterizada como Leishmania braziliensis e o vetor, Lutzomyia intermedia. Foram detectados cães e equídeos infectados por leishmania. O acometimento de crianças menores de 10 anos, o acometimento igual entre os sexos e um componente de agregação familiar sugerem um padrão de transmissão peri ou intradomiciliar.
\end{abstract}

Palavras-chaves: Leishmaniose tegumentar. Surto. Leishmania. Epidemiologia.

Abstract An outbreak of American cutaneous leishmaniasis (ACL) was detected in the village of Canoa in 1993. A prospective observational study was outlined to determine the frequency rates and to clinically characterize the disease. A total of 555 people were followed up. There were 29 cases of ACL, 11 cases of probably previous ACL (scars) and 529 healthy individuals. Of these 529 individuals, 65 had a positive Montenegro reaction without any present or past evidence of leishmaniasis. The prevalence of ACL during the two years was 5.2\% (29/555). The leishmania involved was Leishmania braziliensis and the vector, Lutzomyia intermedia. Evidence of infection was detected in dogs and horses. The high frequency of the disease among children under ten years, the similar sex distribution of cases and a component of familial aggregation suggest a peri- or intra-domiciliary transmission.

Key-words: Cutaneous leishmaniasis. Outbreak. Leishmania. Epidemiology.

A leishmaniose tegumentar já foi descrita em todos os continentes, exceto na Oceania, estimando-se em cerca de 185.380 .000 pessoas sob o risco da infecção, com a ocorrência de 295.900 casos novos anuais sendo 59.300 nas Américas ${ }^{4}$. Nas Américas, a leishmaniose tegumentar americana (LTA) se distribui amplamente, desde o Texas até o norte da Argentina, não havendo relato de casos apenas no Canadá, Chile e Uruguai. No Brasil, a doença tem sido documentada em praticamente todos os estados ${ }^{16} 22$. A LTA é conhecida no estado da Bahia desde o início do século XIX ${ }^{25} 29$. Nessa chamada de fase baiana da doença, a expansão da cultura de cacau atraía trabalhadores de várias regiões do país e a maioria dos pacientes era proveniente do Recôncavo e região sul do estado, áreas servidas pela estrada de ferro ${ }^{8}$.

Serviço de Imunologia do Hospital Universitário Professor Edgar Santos (HUPES) da Universidade Federal da Bahia (UFBA). Apoio financeiro: PRONEX, FINEP e NIH GRANT \#30.639.

Endereço para correspondência: Dr. Edgar M. Carvalho. Departamento de Imunologia/Hospital Universitário Prof. Edgar Santos. R. João das Botas s/nำ, $3^{\circ}$ andar, Canela, 40110-160 Salvador, BA, Brasil. Tel: 55 71 339-6320/237-7353. Fax: 55 71 245-7110.

Recebido para publicação em 19/8/98. 
Atualmente, a LTA é endêmica em praticamente todo o estado, com transmissão ativa e surtos epidêmicos registrados em várias localidades. Em Três Braços, onde desde 1974 existe um núcleo de estudo da LTA, a prevalência é de 14,9\% e a incidência anual média de 8,1 casos $/ 1.000$ habitantes, com $2,7 \%$ evoluindo para a forma mucosa, evolução esta que ocorre em uma média de 6 anos ${ }^{19}$. Vários trabalhos vêm caracterizando os aspectos clínicos e epidemiológicos da LTA na região 56819 , porém poucos estudos clínicoepidemiológicos existem em outras áreas da Bahia $^{26}$

O risco de infecção, classicamente atribuído às formas de ocupação dos ambientes florestais pelo homem (formas zoonóticas), vem ressurgindo com outra feição em áreas onde focos ativos da doença sobreviveram em pequenas matas residuais, havendo a urbanização da LTA (formas antroponóticas), com adaptação dos agentes e vetores aos novos ambientes, envolvendo os animais domésticos ${ }^{23}$. A urbanização das leishmanioses tem sido documentada em diversos estados do Brasil como São Paulo, Rio de Janeiro e Espírito Santo 1516242728 . O papel dos surtos na perpetuação da endemia é conhecido, mas a falta de estudos sobre a estrutura epidemiológica dos mesmos dificulta o conhecimento dos fatores envolvidos na incidência humana dessa doença ${ }^{1516}$.

Em março de 1993 um surto de LTA foi detectado no vilarejo de Canoa, município de Santo Amaro, Bahia, onde não havia registro prévio da doença. Nesse trabalho são descritos os aspectos clínico-epidemiológicos da LTA neste vilarejo.

\section{MATERIAL E MÉTODOS}

Caracterização geográfica da região. O município de Santo Amaro situa-se no Recôncavo Baiano a $71 \mathrm{~km}$ da capital do estado, Salvador, 12 33' latitude sul, 38 42' longitude oeste, $50 \mathrm{~m}$ com relação ao nível do mar. A temperatura média anual é de $25,4^{\circ} \mathrm{C}$. Canoa é um povoado rural, com 163 habitações, distribuídas ao longo de uma estrada vicinal de $2,6 \mathrm{~km}$, na linha de cumeada do centro de dispersão de dois rios, rio Subaé e rio Traripe. Há predomínio de plantações de bananas e mandioca que chegam em alguns trechos a rodear totalmente as casas. Há luz, porém não há água encanada ou rede de esgoto. As casas (Figura 1) são na maioria bem simples,

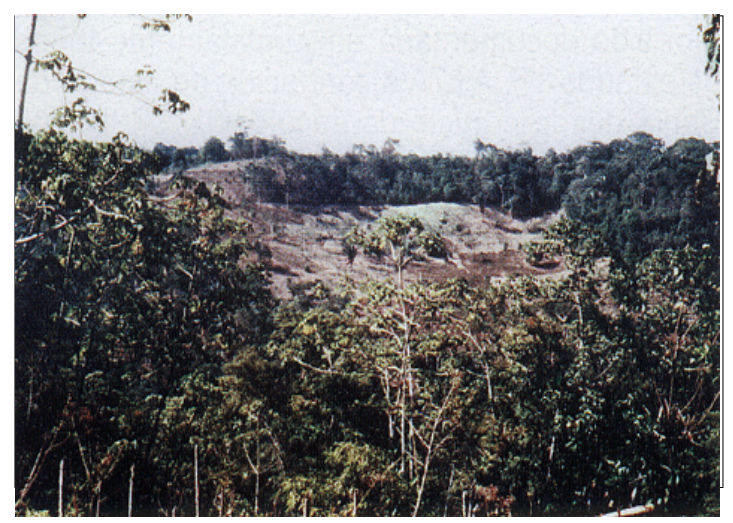

Figura 1- Área de desmatamento recente ao fundo da casa 163, Canoa- Santo Amaro-Bahia. de reboco, teto de telha e chão de cimento. O terço final do povoado, da casa 101 até a casa 163, é denominado Canoa de Baixo e os dois terços iniciais, da casa 001 até a casa 100, denominado de Canoa de Cima. A mata ao redor é resíduo secundário da Mata Atlântica, evidenciando extensas áreas de desmatamentos antigos e recentes (Figura 2), estes últimos predominando no terço final do povoado.

Inquérito epidemiológico. O presente estudo desenvolveu-se de março de 1993 a março de 1995. No primeiro ano do estudo, todas as casas do povoado foram cadastradas, com ficha clínicoepidemiológica por casa, e todos os moradores

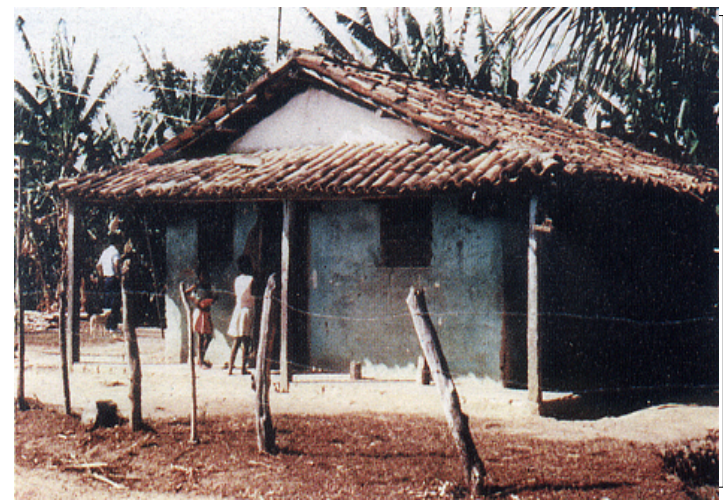

Figura 2 - Casa 163, padrão típico das habitacões do povoado, onde foram detectados 7 casos de LTA. 
avaliados com exame clínico-dermatológico e reação de Montenegro. No decorrer dos dois anos todos os casos suspeitos de LTA cutânea ou mucosa e todos indivíduos que apresentaram reação de Montenegro positiva foram avaliados, clinica e laboratorialmente, inclusive com exame otorrinolaringológico.

Exames laboratoriais. A Reação de Montenegro foi realizada com antígeno solúvel de Leishmania amazonensis (MHOMBr88-Ba 125) padronizado com $250 \mu$ gramas de proteína $/ \mathrm{ml}$. A sorologia para leishmaniose foi realizada pela técnica padrão de ELISA, previamente descrita. A cultura do aspirado da lesão ou do gânglio próximo à lesão foi realizada em meio de cultura NNN. O exame histopatológico foi feito pela técnica de hematoxilina-eosina.

Inquérito nos animais domésticos e inquérito flebotomínico. Dois inquéritos sorológicos caninos foram realizados, um em maio de 1993 e outro em maio de 1994, sendo o primeiro através da técnica de imunofluorescência com sangue colhido em papel de filtro e o segundo através da técnica de ELISA em soro. Um inquérito sorológico nos equídeos foi realizado em outubro de 1994, através da técnica de ELISA, com material processado na Fundação Oswaldo Cruz (FIOCRUZ) do Rio de Janeiro, RJ. Um inquérito flebotomínico foi feito em três pontos diferentes do povoado, através das armadilhas de Shannon e luminosas. A caracterização da espécie de flebotomíneo foi feita no Instituto Oswaldo Cruz (FIOCRUZ) de Salvador, BA.

Análise estatística. Os dados coletados foram inseridos em banco de dados do programa EPIINFO versão 6.0, da Organização Mundial de Saúde. Para análise de significância estatística foram utilizados os seguintes testes: Teste "t" de Student, teste exato de Fisher, qui-quadrado, razão de prevalência e risco relativo. As diferenças foram consideradas estatisticamente significantes quando a probabilidade de erro tipo I foi $<0,05$.

\section{RESULTADOS}

O inquérito populacional clínico-epidemiológico constatou a presença de 163 habitações, com 604 habitantes, dos quais 555 foram avaliados. A média de idade da população foi de $24,0 \pm 19,6$ anos, sendo $283(51 \%)$ do sexo masculino e 306 $(55,2 \%)$ com menos de 20 anos de idade. O número de moradores da área de Canoa de Baixo foi de 202 (36,4\%) indivíduos.

O inquérito sócio-econômico realizado revelou serem os chefes de família predominantemente analfabetos ou com alfabetização básica $(66,4 \%)$ e lavradores $(71,4 \%)$. Com relação aos domicílios, o inquérito revelou que a maioria das habitações era de taipa (56\%), com piso de cimento $(77,5 \%)$, sem água potável $(79,1 \%)$, sem fossa $(79,1 \%)$ e com energia elétrica (94\%). Não houve diferença estatisticamente significante com relação às variáveis sócio-econômicas quando se comparou Canoa de Cima com Canoa de Baixo. Também não houve diferença estatisticamente significante quando se comparam as variáveis sócioeconômicas dos domicílios com casos presentes ou passados de doença com as dos domicílios sem casos de doença.

Foram registrados 129 cães (1,08 cão/ domicílio $\pm 1,46), 61$ gatos $(0,52$ gato/domicílio $\pm 0,81)$ e 135 equídeos (1,13 eqüídeo/domicílio $\pm 1,31$ ) nos domicílios residenciais.

No decorrer do primeiro ano, foram avaliados 555 moradores, encontrando-se na primeira avaliação 11 indivíduos com LTA, 11 com história e cicatriz características de doença passada sem tratamento específico e com o teste intradérmico positivo (cura espontânea) e 533 sadios clinicamente e sem história pregressa de LTA. Neste último grupo, $468(87,8 \%)$ apresentaram reação de Montenegro negativa e 65 (12,2\%) positiva. Ainda no primeiro ano de estudo mais 7 casos de LTA foram diagnosticados e no decorrer do segundo ano mais 11, todos com avaliações prévias negativas. Ao todo ocorreram 29 casos de LTA. A prevalência de LTA com lesões ativas nos dois anos do estudo foi de 5,2\% (29/555). A razão de prevalência da doença ativa em Canoa de Baixo com relação a Canoa de Cima foi de 4,59 [IC95\%; 2,07-10,17]. A prevalência de indivíduos sadios com reação de Montenegro positiva foi de $11,7 \%(65 / 555)$. A incidência de LTA foi de 3,5\% (14/464) na coorte acompanhada.

Características clínicas da leishmaniose tegumentar. Dos 29 casos diagnosticados de LTA, $23(79,3 \%)$ apresentavam forma cutânea, $5(17,2 \%)$ a forma cutâneo-mucosa simultânea e um $(3,4 \%)$ a forma disseminada (também com lesão mucosa). Não ocorreram casos de forma mucosa isolada ou de forma difusa da doença. Dos 29 casos, 21 ocorreram em Canoa de Baixo e 8 em Canoa de Cima $(p<0,001)$. Esses dados estão demonstrados na Tabela 1. 
Tabela 1 - Distribuição dos casos de LTA nas duas partes do povoado, em Canoa- Santo Amaro- Bahia, 1993-1995.

\begin{tabular}{lcccccc}
\hline Parte do povoado & \multicolumn{2}{c}{ População doente } & \multicolumn{2}{c}{ População sadia } & \multicolumn{2}{c}{ Total } \\
& $\mathrm{n}$ & $\%$ & $\mathrm{n}$ & $\%$ & $\mathrm{n}$ & $\%$ \\
\hline Canoa de Baixo & 21 & 10,4 & 181 & 89,6 & 202 & 100,0 \\
Canoa de Cima & 8 & 2,3 & 345 & 97,7 & 353 & 100,0 \\
\hline Total & 29 & 5,2 & 526 & 94,8 & 555 & 100,0 \\
\hline
\end{tabular}

$\left(p=0,001 ; \chi^{2}=17,15\right)$.

Os 29 casos ocorreram em 15 famílias, com a média de 1,93 casos/família, sendo 11 (73,3\%) famílias com um caso e 4 famílias cada uma com 2, 4, 5 e 7 casos respectivamente. Considerandose o fato de existir ou ter existido um doente na casa, a chance de ter outro doente na mesma casa foi 4,5 vezes maior nesses casos do que em casas sem doente $(p<0,0001)$.

Os casos de LTA foram diagnosticados predominantemente(22/29) de dezembro a março de cada ano. Não houve diferença estatisticamente significante dos 29 casos com relação a média de idade e sexo, quando comparados com a população geral. A distribuição dos casos de acordo com a ocupação mostra que $14(48,3 \%)$ eram estudantes e 10 (34,5\%) eram lavradores.

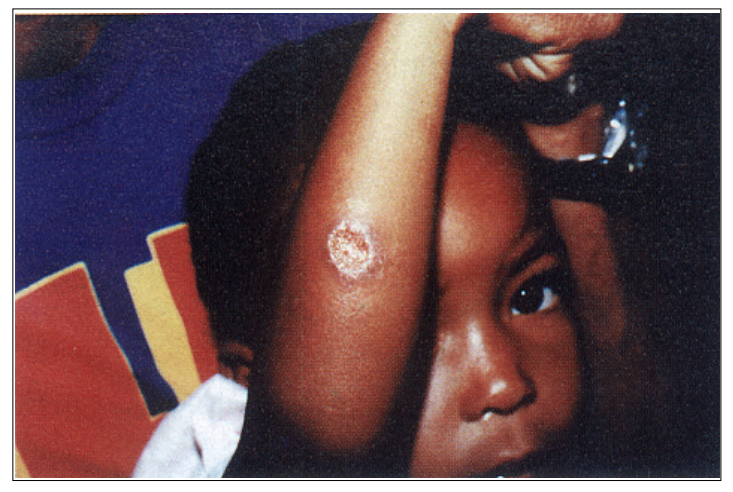

Figura 3- Lesão ulcerada típica em membro superior direito de criança de 3 anos.

tempo de evolução maior que 60 dias e das formas pápulo-nodulares e ectimatosas com um tempo de evolução menor que 60 dias $(p<0,0001)$. $O$ tamanho médio das lesões foi de 18,5 $\pm 9,8 \mathrm{~mm}$. O comprometimento mucoso simultâneo a lesões cutâneas ocorreu em seis $(20,7 \%)$ dos 29 casos.
O número total de lesões foi de 77 lesões para 28 casos, excluindo-se dessa contagem o paciente com a forma disseminada. A média de lesões por paciente foi de 2,8 lesões. Não houve diferença estatisticamente significante na proporção de crianças com múltiplas lesões, quando comparadas com os adultos na mesma situação. A distribuição por local afetado mostra que de 77 lesões, 55 (71,4\%) situavam-se acima da linha da cintura pélvica, não havendo diferença entre crianças e adultos.

A lesão do tipo ulcerada (Figura 3) ocorreu em $45(58,5 \%)$ das 77 lesões e outras formas, pápulo-nodular e ectimatosas (Figura 4), em 32 $(41,5 \%)$ lesões. Associando-se os tipos clínicos com o tempo de evolução, observa-se uma relação direta das formas ulceradas com um

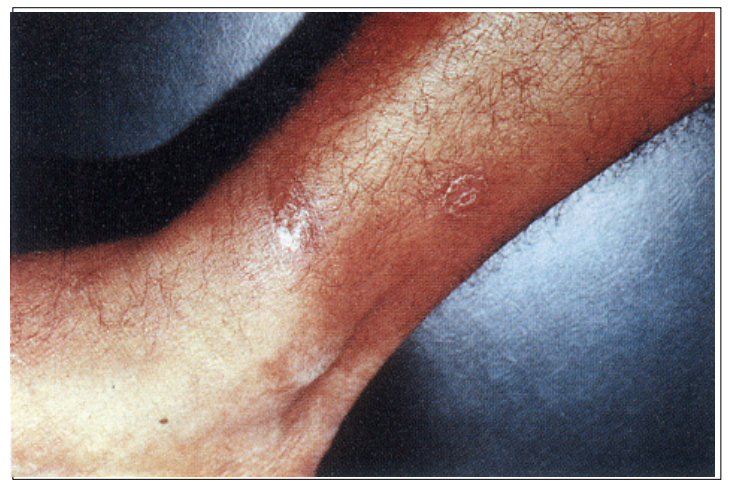

Figura 4- Duas pequenas lesões pápulo-ulceradas em membro inferior direito, com 8 dias de evolução e que ao exame histopatológico apresentavam índice parasitário 3 (100 parasitas $X 100$ campos).

Todos os casos de comprometimento mucoso simultâneo ocorreram com lesões múltiplas e com lesões acima da cintura pélvica $(p<0,0001)$. A adenopatia ocorreu em 23 (79,3\%) dos 29 casos. Nos casos de diagnóstico recente, com tempo de evolução menor ou igual a 60 dias, 
todos apresentaram adenopatia. O comprometimento ganglionar caracterizou-se por ser na cadeia próxima à lesão, em alguns casos bilateralmente, mesmo sem lesão no lado contra lateral (6/23), com características inflamatórias (móveis, dolorosos e não aderidos), chamando atenção, em sete casos, o tamanho volumoso dando aspecto tumoral. Nos casos em que o comprometimento era também contra lateral, os gânglios tinham tamanho maior que $5 \mathrm{~cm}$, eram dolorosos, fusionados e não aderidos.

A reação de Montenegro foi positiva em 26 dos $29(89,7 \%)$ casos na primeira avaliação com lesão suspeita, sendo a média da enduração de $17,6 \pm 1,4 \mathrm{~mm}$. Dois dos doentes com reação de Montenegro inicial negativa, tinham oito e quinze dias de evolução, e tornaram-se positivos quando repetiram a reação. A sorologia (ELISA) para leishmaniose foi positiva em $16(55,2 \%)$ casos ,apresentando uma relação direta com o tempo de evolução maior que 60 dias $(p<0,001)$.

Os achados histopatológicos predominantes foram: presença de intenso infiltrado inflamatório linfo-plasmocitário $(64,7 \%)$, de reação granulomatosa $(76,5 \%)$ e de epidermotropismo $(82,4 \%)$. Em todos os 11 casos, nos quais o parasito foi cultivado, a espécie de leishmania caracterizada através de anticorpo monoclonal foi a Leishmania braziliensis.

Os casos de doença foram tratados com antimonial pentavalente (Glucantime), via venosa, na dose de $20 \mathrm{mg} \mathrm{Sb} / \mathrm{kg} /$ dia durante 20 dias, repetindo-se a série após 4 semanas da primeira naqueles que ainda apresentavam lesão ativa. Não houve caso de falência terapêutica.

Inquérito nos animais domésticos e inquérito flebotomínico. No primeiro inquérito canino, realizado em maio de 1993, foram examinados 104 cães dos quais cinco $(4,8 \%)$ tiveram a sorologia positiva para leishmaniose. No segundo inquérito canino, feito em dezembro de 1994, foram examinados 100 cães dos quais oito $(8 \%)$ tiveram a sorologia positiva para leishmaniose. Em setembro de 1994, o exame clínico e inquérito sorológico foram realizados nos equídeos do povoado, tendo sido examinados 77 animais. Nenhum eqüídeo apresentou lesão cutânea sugestiva de leishmaniose e o teste sorológico foi positivo em 17 (22\%). Em dezembro de 1994, o inquérito flebotomínico realizado evidenciou predomínio da Lutzomyia intermedia em 94\% dos flebótomos examinados (47/50), mas também sendo capturados Lutzomyia migonei e Lutzomyia (Nyssomyia) sp.

\section{DISCUSSÃO}

Mudanças ambientais têm sido associadas a mudanças no perfil epidemiológico da leishmaniose tegumentar, com descrições cada vez mais freqüentes de surtos em áreas urbanas e peri-urbanas, sugerindo uma adaptação do vetor e de todo seu ecossistema a essas regiões ${ }^{13}$. O presente estudo detectou e acompanhou um surto de leishmaniose tegumentar no povoado rural de Canoa, município de Santo Amaro, BA, área de colonização antiga e população estável. A diferença estatisticamente significante encontrada entre o grupo de sadios com o de doentes foi o do local de moradia, havendo no último grupo um forte predomínio de moradores de Canoa de Baixo, terço final do povoado onde uma alteração ambiental muito acentuada vem acontecendo, com desmatamentos recentes e antigos e uma maior proximidade da mata às casas. As demais variáveis testadas não mostraram diferenças, sugerindo que a questão seja ambiental. Os casos de LTA ocorreram predominantemente nos meses de dezembro a março, época de verão, calor e umidade na região. Não existe claramente um padrão sazonal na transmissão da leishmaniose tegumentar e os estudos demonstram grande variação na época de maior ocorrência, havendo relatos de maior predominância do vetor nos meses quentes e úmidos ${ }^{15} 19$.

No presente estudo houve um comprometimento elevado de crianças, com taxas semelhantes entre os dois sexos e com um possível componente de agregação familiar, sugerindo padrão de transmissão peridomiciliar ou intradomiciliar. Este padrão de transmissão é freqüentemente descrito na região Sudeste do país ${ }^{16} 1724$. As lesões eram múltiplas, extensas e com localização predominante acima da cintura pélvica. Esses são fatores de risco ${ }^{21}$ para o desenvolvimento da forma mucosa e justificam a elevada freqüência de doença mucosa observada nesse surto. A ocorrência de múltiplos casos em algumas famílias sugere transmissão peridomiciliar, podendo também se levantar a questão do papel do homem na cadeia de transmissão e possíveis fatores genéticos ${ }^{74}$. Estudos futuros em área de transmissão de L. braziliensis precisam ser desenvolvidos para detectar a importância dos fatores genéticos no comportamento da infecção e da doença.

O achado de lesões predominantemente na porção superior do tronco não é o mais comum 
em áreas de LTA e também sugere transmissão intradomiciliar. Os flebótomos costumam voar baixo e ao atingir pessoas deitadas poderiam alcançar mais facilmente as regiões altas do corpo ${ }^{3}$. Em crianças, pela baixa estatura, é freqüente o acometimento acima da cintura pélvica9 .

O comprometimento ganglionar foi um achado que esteve presente em todos os casos de diagnóstico precoce. A linfadenopatia vem sendo reconhecida na literatura como um achado freqüente e importante nos casos de LTA, principalmente quando de diagnóstico precoce ${ }^{56}$. É possível que o comprometimento ganglionar seja mais habitual do que o relatado em diversas séries, estando associado ao início do desenvolvimento das lesões e que estudos em campo, dentro da área endêmica, tenham mais chances de detecta-lo. A reação de Montenegro é um exame de grande valor para diagnóstico de LTA, considerando ter mais de $90 \%$ de sensibilidade e especificidade nas mais diversas séries ${ }^{14} 18$ 28. Em Canoa, a elevada taxa de positividade da reação de Montenegro reforça a importância do teste intradérmico no diagnóstico das leishmanioses.
A positividade encontrada nos inquéritos sorológicos canino e equídeo revela uma participação dos referidos animais domésticos no ciclo da doença no local, embora o real papel dessa participação necessite de estudos mais específicos. A documentação de cães ${ }^{10} 1120$, equídeos ${ }^{1231}$ e roedores domésticos ${ }^{23}$ infectados por leishmania sugere a participação dos mesmos na domiciliação e urbanização da LTA.

$O$ encontro de $L$. intermedia nos domicílios e peridomicílios, em áreas rurais e peri-urbanas, sugere uma evolução da espécie à domiciliação ${ }^{13}$ 151622 23. Em Três Braços, Bahia o predomínio é de $L$. whitman ${ }^{30}$, com padrão de transmissão ainda associado a áreas de florestas e ocasionalmente ocorrendo em áreas peridomiciliares ${ }^{19}$. Em Jequié $^{26}$, Bahia, os dados também indicam um padrão relacionado à maior exposição humana à floresta. O predomínio de $L$. intermedia em Canoa bem como as outras características do surto que indicam transmissão peri ou intradomiciliar diferem até então do padrão epidemiológico de LTA encontrado no Estado da Bahia.

\section{AGRADECIMENTOS}

Agradecemos ao Dr. Sebastião Dias e toda equipe da Santa Casa de Misericórdia de Oliveira dos Campinhos, onde os pacientes foram acompanhados e tratados; à Fundação Nacional de Saúde (FNS) pelo apoio em todas etapas do estudo, especificamente aos Sr. Benedito Barbosa e Sr. Almir H. Adorno Neto; ao Dr. Hélio Lessa pelos exames otorrinolaringológicos; ao Dr. Roque Almeida pelo preparo dos antígenos para reação de Montenegro.

\section{REFERÊNCIAS BIBLIOGRÁFICAS}

1. Aguilar CM, Fernandez E, Fernandez R, Deane LM. Study of an outbreak of cutaneous leishmaniasis in Venezuela: the role of domestic animals. Memórias do Instituto Oswaldo Cruz 79: 181- 195, 1984.

2. Alencar EJ, Pessoa EP, Fontenele ZF. Infecção natural de Rattus rattus alexandrinus por Leishmania (Provavelmente $L$. braziliensis) em zona endêmica de leishmaniose tegumentar do Estado do Ceará, Brasil. Revista do Instituto de Medicina Tropical de São Paulo 2:347-348, 1960.

3. Araújo-Filho NA, Coura JR, Reis VLL. Leishmaniose tegumentar americana na Ilha Grande, Rio de Janeiro. III. Reservatórios silvestres e comensais. Revista do Instituto de Medicina Tropical de São Paulo 14:153-161, 1981.

4. Ashford RW, Desjeux P, Raadt P. Estimation of Risk of Infection and Number of cases of Leishmaniasis. Parasitology Today 3:104-105, 1992.

5. Barral A, Barral-Netto M, Almeida R, Jesus AR, Grimaldi Jr GJ, Netto EM, Santos I, Bacellar O, Carvalho EM.
Linphadenopathy associated with Leishmania braziliensis cutaneous infection. Americam Journal of Tropical Medicine and Hygiene 47:587-592, 1992.

6. Barral A, Guerreiro J, Bonfim G, Correia D, Barral-Netto $M$, Carvalho EM. Linphadenopathy as the first sign of human cutaneos infection by Leishmania braziliensis. American Journal of Tropical Medicine and Hygiene 53:256-259, 1995.

7. Cabello PH, Lima AMVMD, Azevedo ES, Krieger $\mathrm{H}$. Familial aggregation of Leishmania chagasi infection in northeastern Brazil. American Journal of Tropical Medicine and Higyene 52:364-365, 1995.

8. Costa JML, Tada MS, Martins- Netto EM, Vale KC, Lago EL, Marsden PD. Procedência de pacientes portadores de leishmaniose tegumentar americana nas áreas endêmicas de Três Braços e Corte de Pedra- Estado da Bahia- Brasil. Revista da Sociedade Brasileira de Medicina Tropical 21: 145-149, 1988.

9. Cucé LC, Oliveira ZNP, Belda Júnior W, Zolli CA. Leishmaniose tegumentar americana na infância- 
Aspectos epidemiológicos, clínicos e terapêuticos. Anais Brasileiros de Dermatologia 65:18S-19S, 1990.

10. Falqueto A, Coura JR, Barros GC, Grimaldi-Filho G, Sessa PA, Carias VR, Jesus AC, Alencar JJA. Participação do cão no ciclo de transmissão de leishmaniose tegumentar no município de Viana, Estado do Espírito Santo, Brasil. Memórias do Instituto Oswaldo Cruz 81:155-163, 1986.

11. Falqueto $A$, Sessa PA, Varejão JBM, Barros GC, Momen $\mathrm{H}$, Grimaldi Jr G. Leishmaniasis due to Leishmania braziliensis in Espírito Santo State, Brazil. Further evidence on the role of dogs as a reservoir of infection for humans. Memórias do Instituto Oswaldo Cruz 86:499$500,1991$.

12. Falqueto A, Varejão JBM, Sessa PA. Cutaneous leishmaniasis in a horse (Equus caballus) from endemic area in the state of Espírito Santo, Brazil. Memórias do Instituto Oswaldo Cruz, Rio de Janeiro 82:443, 1987.

13. Forattini OP, Rabello EX, Serra OP, Cotrim MD, Galati EAB, Barata JMS. Observações sobre a transmissão da leishmaniose tegumentar no Estado de São Paulo, Brasil. Revista de Saúde Pública, São Paulo 10:31-43, 1976.

14. Furtado T. Critérios para diagnóstico da leishmaniose tegumentar americana. Anais Brasileiros de Dermatologia 65:51-86, 1980

15. Gomes AC. Aspectos epidemiológicos sobre a transmissão da leishmaniose tegumentar na Região do Vale do Ribeira, Estado de São Paulo, Brasil. São Paulo. Tese de Livre Docência - Faculdade de Saúde Pública USP, 1985.

16. Gomes AC. Perfil epidemiológico da Leishmaniose Tegumentar no Brasil. Anais Brasileiros de Dermatologia 67:55-60, 1992.

17. Gomes AC, Yamamoto YI, Capinzaiki AN, Amaral NMM, Guimarães AJG. Aspectos ecológicos da leishmaniose tegumentar americana. 9- Prevalência/incidência da infecção humana nos municípios de Pedro de Toledo e Miracatu, São Paulo, Brasil. Revista do Instituto de Medicina Tropical de São Paulo 34:149-158, 1992.

18. Guedes ACM, Cucé LC, Furtado T. Avaliação imunológica e histopatológica de reação de Montenegro. Anais Brasileiros de Dermatologia 65:34S-40S, 1990.

19. Jones TC, Johonson WD, Barreto AC, Lago E, Badaró R, Cerf B, Reed SG, Martins Netto E, Tada MS, Franca F, Wiese K, Golightly L, Frikrig E, Costa JML, Cuba CAC, Marsden PD. Epidemiology of american cutaneous leishmaniasis due to Leishmania braziliensis braziliensis. The Journal of Infectous Diseases 156:73-83, 1987.
20. Lainson R, Shaw JJ. The role of animals in the epidemiology of South American leishmaniasis. In: Lumsden WHR, Evans DA (eds) Biology of the Kinetoplastida. London, Academic Press 1:116, 1979

21. Marsden PD. Mucosal leishmaniasis ("espundia" Escomel, 1911). Transactions of the Royal Society of Tropical Medicine and Hygiene 80:859-876, 1986.

22. Marzochi MCA. A Leishmaniose Tegumentar no Brasil. In: Grandes Endemias Brasileiras. Brasília, Editora da Universidade de Brasília, 1989.

23. Marzochi MCA, Marzochi KBF. Tegumentary and visceral leishmaniasis in Brazil- Emerging anthropozoonosis and possibilities for their control. Cadernos de Saúde Pública, Rio de Janeiro, 10:359-375, 1994 .

24. Mayrink W, Williams $P$, Coelho MV, Dias M, Martins AV, Magalhães PA, Da Costa CA, Falcão AR, Melo MN, Falcão AL. Epidemiology of dermal leishmaniasis in the rio Doce Valley. State of Minas Gerais, Brasil. Annals of Tropical Medicine and Parasitology 73:123-137, 1979.

25. Silva P. La leishmaniose cutânea à Bahia. Revista Médica 15:275-281, 1912.

26. Santos AJO Nascimento EG, Silva MP, Carvalho LCP. Report on a visceral and cutaneous focus in the town of Jequié, state of Bahia, Brazil. Revista do Instituto de Medicina Tropical de São Paulo 35:583-584, 1993.

27. Sessa PA, Barros GC, Mattos EA, Carias VRD, Alencar JTA, Delmaestro D, Coelho CC, Falqueto A. Distribuição geográfica da L.T.A. no estado do Espírito Santo- Brasil. Revista da Sociedade Brasileira de Medicina Tropical 18:237-241, 1985.

28. Sessa PA, Falqueto A, Barros GCB, Varejão JBM. Resultados da reação de Montenegro em pacientes com leishmaniose tegumentar americana, autócnes do Estado do Espírito Santo. Revista da Associação Médica Brasileira 37:115-118, 1991.

29. Torres O. A leishmaniose na Bahia. Arquivos Brasileiros de Medicina.7:374-425, 1920.

30. Vexanat JA, Barreto AC, Cuba CAC, Marsden PD. Características epidemiológicas da leishmaniose tegumentar americana em uma região endêmica do Estado da Bahia. III. Fauna flebotomínica. Memorias do Instituto Oswaldo Cruz 81:293-301, 1986.

31. Vexenat TA, Barreto AC, Rosa AC, Salles CC, Magalhães AV. Infecção natural de Equus asinus por Leishmania braziliensis braziliensis, Bahia, Brasil. Memórias do Instituto Oswaldo Cruz, Rio de Janeiro 8:237-238, 1986. 